\title{
Generating Ontologies from Relational Data with Fuzzy-Syllogistic Reasoning
}

\author{
Bora İ. Kumova ${ }^{(\bowtie)}$ \\ Department of Computer Engineering, \\ İzmir Institute of Technology, 35430 Turkey \\ borakumova@iyte.edu.tr
}

\begin{abstract}
Existing standards for crisp description logics facilitate information exchange between systems that reason with crisp ontologies. Applications with probabilistic or possibilistic extensions of ontologies and reasoners promise to capture more information, because they can deal with more uncertainties or vagueness of information. However, since there are no standards for either extension, information exchange between such applications is not generic. Fuzzy-syllogistic reasoning with the fuzzy-syllogistic system ${ }^{4} \mathrm{~S}$ provides 2048 possible fuzzy inference schema for every possible triple concept relationship of an ontology. Since the inference schema are the result of all possible set-theoretic relationships between three sets with three out of 8 possible fuzzy-quantifiers, the whole set of 2048 possible fuzzy inferences can be used as one generic fuzzy reasoner for quantified ontologies. In that sense, a fuzzy syllogistic reasoner can be employed as a generic reasoner that combines possibilistic inferencing with probabilistic ontologies, thus facilitating knowledge exchange between ontology applications of different domains as well as information fusion over them.
\end{abstract}

Keywords: Relational data · Ontology learning · Syllogistic reasoning · Fuzzy logic

\section{Introduction}

Relational modelling facilitates maintaining data consistency, whereas ontological modelling facilitates logical reasoning with the data [30]. Since most data of every enterprise is usually maintain in relational models, there is increasing demand for automating reasoning with the relational data, in order to further utilise the information systems as decision support systems (Fig. 1). The objective of this paper is to review state-of-the-art in ontology learning and reasoning and to suggest a common possibilistic reasoner for probabilistic and quantified ontologies.

A variety of approaches for ontology learning have been proposed, ranging from unstructured data, like internet text search results, over semi structured data, like partially normalised data, to structured ones, like object-oriented data $[29,51]$ or normalised relational data $[37,20]$. Here we will focus on relational data only. 
In most approaches, first an ontology is generated manually for a given relational data schema, thereafter the ontology is used in conjunction with the relational schema for reasoning with the data [18].

Ones an ontology is available for a particular relational data schema, an appropriate ontology reasoner can be chosen, which will then enable reasoning over the relational data, via the ontology. While an ontology is usually stored separately in a file or database, a reasoner is part of the semantic web application.

It is widely accepted that uncertainties of a domain that find reflections in the relational data, can be represented with related probabilities within ontologies.

Reasoners for probabilistic ontologies are mostly based on Bayesian networks. If data about such probabilities is unavailable, fuzzy ontologies $[42,8,5]$ or possibilistic $^{1}$ ontologies [34] and reasoners may be preferred instead for processing vague information [28].



Fig. 1. Utilising relational data sources in the semantic web

The lack of standards for ontologies with probabilist or possibilistic extensions, prevent from efficient knowledge exchange between semantic web applications (Fig. 1) that have such extensions.

Here we propose the fuzzy-syllogistic (FS) reasoning system as a common logic for ontology reasoning. The 2048 syllogistic moods of the system cover any possible inference for any transitive concept relationship of the ontology [49]. Every such syllogistic inference has a fixed truth ratio [23], which can however change relative to the cardinalities of the probabilistic samples that make up the concepts and their relationships within the probabilistic ontology. A sample design for FS reasoning with an ontologies that was learned from text was presented elsewhere [49].

\footnotetext{
${ }^{1}$ In this work we will use the term "possibilistic" as a generic term that includes possibilistic logics as well as fuzzy logic, since fuzzy-syllogistic systems cover both.
} 
Reasoning with fuzzy quantifiers or intermediate quantifiers is referred to as approximate reasoning [47]. Fuzzy syllogism refers to individual syllogistic moods that have fuzzy quantifiers [48]. Whereas fuzzy syllogistic system refers to the whole set of all 256 syllogistic moods, along with the truth ratio of every individual mood [24], where moods may have classical quantifiers or fuzzy quantifiers [22].

The paper is organised as follows. First the literature on ontology learning and reasoning with probabilistic and possibilistic approaches is reviewed, thereafter FS reasoning for ontology learning and reasoning is proposed as common reasoner for quantified ontologies with probabilist and possibilistic extensions.

\section{Ontology Learning and Reasoning}

Before we review approaches for learning ontologies, particularly database ontologies and approaches for reasoning with them, we briefly explain ontologies, database ontologies and how database ontologies can be compiled from databases.

\subsection{Ontologies}

An ontology is an object-oriented conceptualisation of a particular domain, such that it allows for logical reasoning about the domain. It is used to specify and share a domain in a common language. Ontologies are always attached with uncertainties, whether they emerge from probabilistic data or are created from possibilistic knowledge, but such extensions are not always reflected on the ontology.

An ontology consists of the following primitives [29]:

- Domain objects: Classes and instances.

- Object attributes: Aspects, properties, features, characteristics, parameters.

- Object relationships: Qualitative or quantitative relations between attributes.

- Processes \& events involving objects: Functionalities modifying attribute values.

- Logic: Rules for valid reasoning information from attribute values.

- Uncertainties: Probabilities attached to anyone of the above primitives.

- Vagueness: Possibilities attached to anyone of the above primitives.

An ontology usually does not store or reference samples for any of its primitives, but could be extended with such a capability. Such an extension for database ontologies [27] is discussed below.

\section{Database Ontologies}

The relational database model is a derivation from first-order predicate logic [12]. However, there are no reasoners available for databases. Since ontologies are also based on first-order predicate logic, the primitives of the different models can be transformed into each other, based on common logical concepts. For instance, 
valid column values of a database table can be specified with predicate-logical quantifiers, which is analogous to valid values of an attribute of an ontology class (table 1).

Table 1. Mapping between relational data concepts and ontological concepts

\begin{tabular}{|c|c|}
\hline Relational & Ontological \\
\hline Entity Table & Class \\
\hline Relation Table & Class Relationship \\
\hline 1-n Relationship & Superclass-Subclass Relationship \\
\hline n-n Relationship & + \\
\hline View $^{+}$ & Multiple Superclass-Subclass Relationships \\
\hline Foreign Key & Process \\
\hline Primary Key & Attribute \\
\hline Attribute & Attribute \\
\hline Attribute Value & Attribute Value \\
\hline Row & Class Instance \\
\hline Not Null; Unique & Cardinality Constraints \\
\hline
\end{tabular}

\subsection{Compiling Database Ontologies}

Formal transformations between the models are one way for generating database ontologies.

Transformations could be performed principally bidirectional, if formal definitions for mapping the elements between the two models [26] are available. Whereby the time complexity of such mapping algorithms is usually linear [26]. However, since most solutions transform unidirectional, from database to ontology, we prefer the term compiling.

Two major approaches are distinguished for compiling an ontology from a database, reverse engineering and schema transformation. The former transform relational schema into ontological schema $[18,44,21,1,26]$, whereas the latter transform entity-relationship (ER) schema into ontology schema $[45,15,32]$. The latter has the advantage that the generated ontology can be used for performing relational database operations via the ontology [27]. Even transformations of fuzzy extended ER into fuzzy ontologies are proposed [50].

In such systems the ontology can be used for strategic decisions using the data concept relationships, while the database is used for retrieving sample data that supports these decisions [38]. Ontological decision making is discussed below under reasoning with ontologies.

\subsection{Learning Database Ontologies}

Learning an ontology is achieved by conceptualising objects of the application domain, their attributes and relationships. This can be accomplished with 
probabilistic approaches that can identify regularities in the data. Therefore, probabilistic ontologies are the result of logical evaluations of domain statistics.

There are various approaches for learning database ontologies [20]. We will focus on approaches that work at least partially automated on the database and that learn either crisp, probabilistic or possibilistic database ontologies (table 2).

Table 2. Tools for ontology learning from relational models

\begin{tabular}{|c|c|c|}
\hline Ontology Learning & Source Data Model & Ontology Logic \\
\hline RDBToOnto $[10]$ & $\mathrm{ER}^{\$}$ Schema \& Relational Schema & Crisp \\
\hline MASTRO $[14]$ & ER Schema & Crisp \\
\hline PROGNOS $[13]$ & Relational Schema & Probabilistic \\
\hline SoftFacts $[43]$ & Relational Schema & Fuzzy \\
\hline
\end{tabular}

\$ER: Entity-Relationship

Some approaches do not generate an ontology, but aim at learning probabilistic models from large relational data [17]. Popular probabilistic approaches are mostly based on Bayesian updating and inferencing, like Multi-Entity Bayesian Networks (MEBN) [25], for learning from relational data [33].

Data mining is rich on techniques for learning regularity of a data set that may be structured or unstructured. Some of these techniques have been adapted to learning database ontologies too. RTAXON is such a learning technique that transforms statistically identified relational data into ontological relationships [11]. It takes both as input, the relational schema and the entity-relationship schema, and can discover data relations not specified in either schema, but found in the data [10].

Interesting is further a data mining example for learning fuzzy ontologies from unstructured data of a hyper-media database that can learn time-varying dynamics of the domain. The adaptive ontology is used in return, to adapt querying the dynamically changing database [7].

\subsection{Reasoning with Ontologies}

Formal systems have the disadvantage of having no learning capability. However they have the advantage that they can be axiomatised and formally checked for consistency, satisfiability, subsumption or redundancy. Such formal methods have to be part of any reasoner [28]. Any ontology, whether specified, compiled or learned, needs to be validated formally, in order to be acceptable as common knowledge.

Inferencing is rule execution in propositional logic. An inference engine is an implementation of the logic, with the objective to execute rules of the knowledge base according to given inference rules, ie resolving rules in forward or backward chaining. The engine may additionally use utility functions, like the Rete algorithm [16], for improving efficiency of rule searching (Fig. 2). 


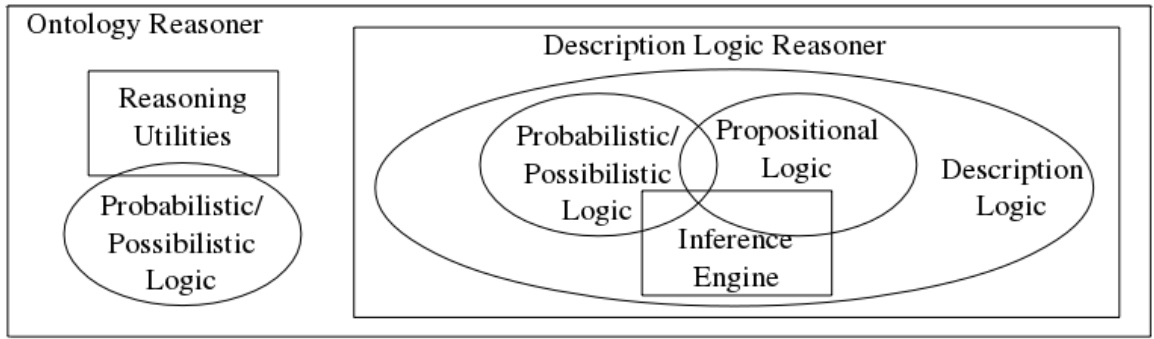

Fig. 2. Probabilistic/possibilistic extensions of logics used in ontology reasoners

\section{Description Logic Reasoners}

Most description logic reasoners use existing inference engines and utilise further techniques, such as decision tree, tableau algorithm or subsumption hierarchy, for handling specifics of description-logical (table 3).

\section{Ontology Reasoners}

An ontology reasoner is an implementation of a description logic reasoner for a particular domain ontology. Both reasoners may be extended separately with probabilistic or possibilistic information (Fig. 2).

For instance, the fuzzy ontology reasoner HyFOM extends the Fuzzy DL further with Mamdani inferencing [46]. Likewise SoftFacts extends Pellet and Fuzzy DL further with fuzzy database query features [43] (table 3).

Table 3. Ontology reasoners and underlying logics

\begin{tabular}{|c|c|c|c|}
\hline Ontology Reasoner & Ontology Logic & Reasoning Logic $\#$ & DL Reasoner \\
\hline MASTRO [14] & Crisp & - & Presto [36] \\
\hline$M E B N[25]$ & Probabilistic & Bayesian & MEBN \\
\hline$B U N D L E[35]$ & Probabilistic & Probabilistic & Pellet $^{+}[39]$ \\
\hline HermiT [31] & Fuzzy & Hyper-Tableau [31] & HermiT [31] \\
\hline FIRE [41] & Fuzzy & - & Fuzzy DL [40] \\
\hline FuzzyDL [3] & Fuzzy & $\begin{array}{l}\text { Fuzzy Rough Sets; } \\
\text { Fuzzy; Lukasiewicz [5] }\end{array}$ & Pellet \\
\hline DeLorean [2] & Fuzzy & Zadeh, Gödel Fuzzy Operators & Pellet \\
\hline HyFOM [46] & Fuzzy & Mamdani & Fuzzy DL \\
\hline SoftFacts [43] & Fuzzy DB & Fuzzy & Pellet \\
\hline$K A O N[34]$ & Possibilistic & Possibilistic & Possibilistic \\
\hline
\end{tabular}

\# Ontology reasoners extended DL reasoners to a specific reasoning logic

${ }^{+}$Pellet implements tableau reasoning 


\section{Applications}

Some of the above discussed ontology learning and reasoning approaches have been implemented as plug-in extensions for ontology development tools, such as Protégé [19] or KAON [34]. Fuzzy Protg is such a sample plug-in [19].

It turns out that only a few applications can learn database ontologies and reason with them probabilistically or possibilistically (table 4). We require these capabilities for our further extension of such systems with FS reasoning.

Table 4. Sample applications that use uncertain ontology learning or reasoning

\begin{tabular}{|c|c|c|c|}
\hline Application & Originality & \multicolumn{2}{|c|}{ Ontology } \\
\cline { 3 - 4 } & & Learning Tool & Reasoning Tool \\
\hline PROGNOS [13] & Knowledge Fusion & MEBN [25] & MEBN [25] \\
\hline UnBBayes [9] & Knowledge Fusion & MEBN [25] & MEBN [25] \\
\hline FEER2FOnto [50] & Semantics-Preserving & Probabilistic & DeLorean [2] \\
\hline
\end{tabular}

Multi-Entity Bayesian Networks (MEBN) [25] extends first-order propositional ontologies with probabilistic information and infers within Bayesian networks of those probability distributions. Whereby the probability distributions are learned again Bayesian. Thus applications that use MEBN can learn and reason probabilistically. PROGNOS [13] and UnBBayes [9] use MEBN for knowledge fusion over relational databases.

FEER2FOnto [50] is interesting in that it combines probabilist learning and fuzzy reasoning with the reasoner DeLorean [2].

\section{Fuzzy-Syllogistic Reasoning}

Fuzzy-syllogistic (FS) reasoning is based on a fuzzy-logical extension of the syllogistic system that consists of all possible combinations of the well known categorical syllogisms. We interpret the system as one complex approximate reasoner that consists of all possible fuzzy-inferences for any given triple concept of an ontology. Here we introduce the fuzzy-syllogistic system ${ }^{4} \mathrm{~S}$ that consists of four affirmative and four negative quantifiers.

\subsection{Fuzzy-Syllogistic System}

A categorical syllogism $\Psi_{1} \Psi_{2} \Psi_{3} F$ is an inference schema that concludes a quantified proposition $\Phi_{3}=S \Psi_{3} P$ from the transitive relationship of two given quantified proportions $\Phi_{1}=\left\{M \Psi_{1} P, P \Psi_{1} M\right\}$ and $\Phi_{2}=\left\{S \Psi_{2} M, M \Psi_{2} S\right\}$ :

$$
\Psi_{1} \Psi_{2} \Psi_{3} F=\left(\Phi_{1}=M \Psi_{1} P, P \Psi_{1} M, \Phi_{2}=S \Psi_{2} M, M \Psi_{2} S, \Phi_{3}=S \Psi_{3} P\right)
$$


where $\mathrm{F}=\{1,2,3,4\}$ identifies the four possible combinations of $\Phi_{1}$ with $\Phi_{2}$, namely syllogistic figures and $\Psi=\left\{\mathrm{A}=\right.$ all; ${ }^{3} \mathrm{I}=$ most; ${ }^{2} \mathrm{I}=$ half; ${ }^{1} \mathrm{I}=$ several; $\mathrm{A}=$ allNot; ${ }^{3} \mathrm{I}=$ mostNot; ${ }^{2} \mathrm{I}=$ halfNot; ${ }^{1} \mathrm{I}=$ severalNot $\}$ are the fuzzy quantifiers. Every syllogistic figure produces $8^{3}=512$ permutations, which are called fuzzysyllogistic moods. Thus the whole system ${ }^{4} \mathrm{~S}$ has in total 2048 fuzzy-syllogistic moods.

Every mood has a structurally fixed truth ratio in $[0,1]$, which is calculated algorithmically [24] by relating the number of its true cases to the number of false cases [23] (table 5 shows sample inferencing in the system $\mathrm{S}$, since these are easier to follow manually). Moods of the FS system become inferences in FS reasoning.

Table 5. Sample fuzzy-syllogistic moods, their truth cases, truth ratios and sample interpretations of the fuzzy-syllogistic system $\mathrm{S}$

\begin{tabular}{|c|c|c|}
\hline $\operatorname{Mood} \Psi_{1} \Psi_{2} \Psi_{3} F$ & $A A A 1, A A I 1$ & $E E I 1,2,3,4$ \\
\hline Cases $\Delta_{i}^{\#}$ & t: 0100101 & $\begin{array}{l}\text { t: } 0110010 ; \text { t: } 1010010 \\
\text { t: } 1110010 ; \text { f: } 1110000\end{array}$ \\
\hline Truth Ratio $\tau$ & $1 \mathrm{t} /(1 \mathrm{t}+0 \mathrm{f})=1.0^{+}$ & $3 \mathrm{t} /(3 \mathrm{t}+1 \mathrm{f})=0.75$ \\
\hline $\begin{array}{l}\text { Interpretation of } \\
\text { false cases* }\end{array}$ & - & At least $P \cap S \neq \Phi$ is missing \\
\hline Example & $\begin{array}{c}\text { All primates are mammals } \\
\text { All humans are primates } \\
\{\text { All, Some }\} \text { humans are mammals }\end{array}$ & $\begin{array}{c}\text { Not All are }\{\text { Turks, Muslim\} } \\
\text { Not All are }\{\text { Orientals, Turks }\} \\
\text { Some Orientals are Muslim }\end{array}$ \\
\hline $\begin{array}{c}\text { Interpretation of } \\
\text { Example }\end{array}$ & $\begin{array}{l}\text { Concluding with All is true, } \\
\text { probably without exception; } \\
\text { concluding with Some is true only } \\
\text { for the possible All case in Some }\end{array}$ & $\begin{array}{l}\text { All four examples that can be } \\
\text { loaded into the four moods are } \\
\text { possibly more true than false, } \\
\text { however possibly not fully true }\end{array}$ \\
\hline
\end{tabular}

${ }^{+} \mathrm{t}=$ true case; $\mathrm{f}=$ false case

* The conclusions of the examples assume that $P \cap S \neq \Phi$ equals the truth ratio $\tau$ of the mood

\# $\Delta_{i}$ syllogistic case $\mathrm{i}=[1,96]$; all possible distinct space permutations of the possible 7 spaces of three sets

\section{$4.2 \quad$ Fuzzy-Syllogistic Learning and Reasoning}

The objective of FS reasoning is to find the best matching fuzzy-syllogistic inference for a given triple concept with transitive relationships. The objective of FS reasoning within the learning process is to accumulate the samples for all possible 7 spaces of three sets, since their 96 possible distinct permutations constitute the universal set, from which the fuzzy-syllogistic moods match some as true syllogistic case and some as false [22]. The outcome of learning is an ontology with quantified relationships.

The FS reasoner calculates for every triple concept with transitive relationships a truth ratio using the very same algorithm for calculating the truth ratios of the individual moods/inferences [22]. In case of learning a fuzzy-syllogistic 
database ontology (Fig. 3) data quantities can easily be determined from the related tables.

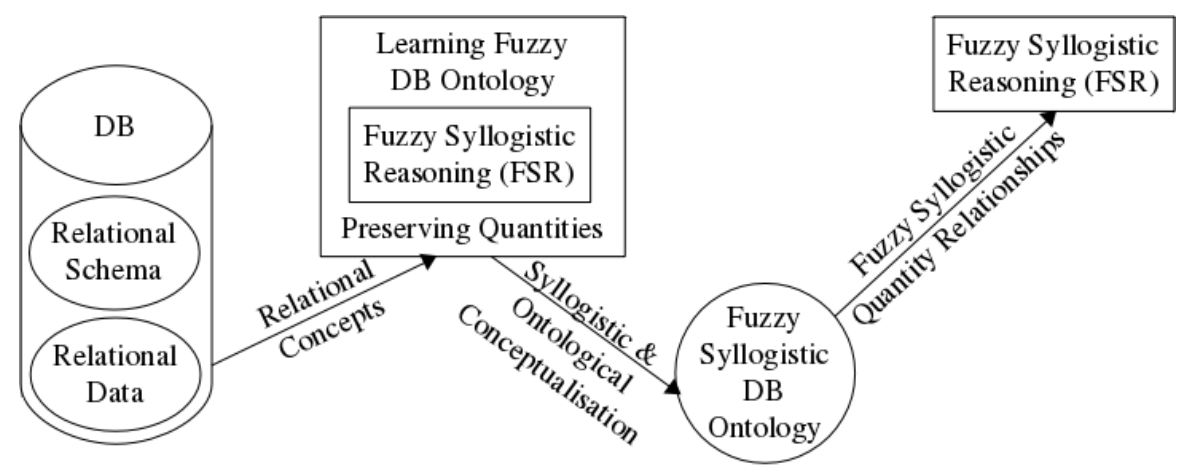

Fig. 3. Learning fuzzy syllogistic ontologies from relational databases using fuzzy syllogistic reasoning

\section{Conclusion}

We have reviewed state-of-the-art in ontology learning and reasoning, with an emphasis on database ontologies. We have pointed out that there are no standards for probabilist or possibilistic extensions of such system and therefore knowledge exchange between them is currently not efficient. Based on these observations, we have suggested fuzzy-syllogistic (FS) reasoning as a common logic for both, learning FS ontologies and reasoning with them. Principally any probabilist learning approach can be extended with FS reasoning, provided that all quantities of the data samples that lead to ontological concepts and their relationships can be calculated during the learning process and remain available along with the FS ontology after learning.

We have briefly discussed FS reasoning using the FS system ${ }^{4} \mathrm{~S}$ that consists of 4 affirmative and 4 negative quantifiers.

Since FS reasoning is based on clear FS systems like, $\mathrm{S}$ or ${ }^{4} \mathrm{~S}$, it could be used as a common possibilistic reasoner for probabilistic ontologies, thus facilitate knowledge exchange in the semantic web. The reasoner can further adjust itself to changing quantities of the domain data, by applying the most suitable FS system ${ }^{n} \mathrm{~S}$ to every triple concept relationship individually. With the FS reasoner individual optimisations are not required, like with fuzzy aggregation operators [6] or fuzzy integrals [4]. 


\section{References}

1. Albarrak, M.K., Sibley, E.H.: Translating relational and object-relational database models into owl model. In: IEEE IRI (2009)

2. Bobillo, F., Delgado, M., Gomez-Romero, J.: DeLorean: a reasoner for fuzzy OWL 1.1. In: Uncertainty Reasoning for the Semantic Web (URSW). LNAI. Springer (2008)

3. Bobillo, F., Straccia, U.: fuzzyDL: an expressive fuzzy description logic reasoner. In: Fuzzy Systems (FUZZIEEE). IEEE Computer Society (2008)

4. Bobillo, F., Straccia, U.: Fuzzy ontologies and fuzzy integrals. In: Intelligent Systems Design and Applications (ISDA). Springer (2011)

5. Bobillo, F., Straccia, U.: Reasoning with the finitely many-valued łukasiewicz fuzzy description logic sroiq. Information Sciences 181 (2011)

6. Bobillo, F., Straccia, U.: Aggregation operators for fuzzy ontologies. Applied Soft Computing 13 (2013)

7. Calegari, S., Loregian, M.: Using dynamic fuzzy ontologies to understand creative environments. In: Larsen, H.L., Pasi, G., Ortiz-Arroyo, D., Andreasen, T., Christiansen, H. (eds.) FQAS 2006. LNCS (LNAI), vol. 4027, pp. 404-415. Springer, Heidelberg (2006)

8. Calegari, S., Ciucci, D.: Fuzzy ontology, fuzzy description logics and fuzzy-OWL. In: Masulli, F., Mitra, S., Pasi, G. (eds.) WILF 2007. LNCS (LNAI), vol. 4578, pp. 118-126. Springer, Heidelberg (2007)

9. Carvalho, R., Laskey, K., da Costa, P.C.G., Ladeira, M., Santos, L., Matsumoto, S.: Unbbayes: Modeling uncertainty for plausible reasoning in the semantic web. In: Semantic Web. Intech (2010)

10. Cerbah, F.: Learning highly structured semantic repositories from relational databases: The RDBToOnto tool. In: Bechhofer, S., Hauswirth, M., Hoffmann, J., Koubarakis, M. (eds.) ESWC 2008. LNCS, vol. 5021, pp. 777-781. Springer, Heidelberg (2008)

11. Cerbah, F.: Mining the content of relational database to learn ontology with deeper taxonomies. In: Web Intelligence and Intelligent Agent Technology, IEEE, WIC (2008)

12. Codd, E.F.: Further normalization of the data base relational model. IBM Reaserch Report (1972)

13. da Costa, P.C.G., Laskey, K.B., Chang, K.C.: PROGNOS: Applying probabilistic ontologies to distributed predictive situation assessment in naval operations. In: International Command and Control Research and Technology Symposium (ICCRTS), C2 Journal (2009)

14. De Giacomo, G., Lembo, D., Lenzerini, M., Poggi, A., Rosati, R., Ruzzi, M., Savo, D.F.: Mastro: A reasoner for effective ontology based data access. In: OWL Reasoner Evaluation Workshop (ORE) (2012)

15. Fisher, M., Dean, M.: Automapper: Relational database semantic translation using OWL and SWRL. OWL experiences and Directions (OWLED), World Wide Web Consortium (w3c) (2008)

16. Forgy, C.: Rete: A fast algorithm for the many pattern/many object pattern match problem. Artificial Intelligence 19 (1982)

17. Getoor, L., Taskar, B.: Introduction to statistical relational learning. MIT Press (2007)

18. Ghawi, R., Cullot, N.: Database-to-ontology mapping generation for semantic interoperability. In: Very Large Databases (VLDB). ACM (2007) 
19. Ghorbel, H., Bahri, A., Bouaziz, R.: Fuzzy protégé for fuzzy ontology models. In: International Protégé Conference (IPC), Stanford Medical Informatics (2009)

20. Hazman, M., El-Beltagy, S.R., Rafea, A.: A survey of ontology learning approaches. International Journal of Computer Applications 22(9) (2011)

21. He-ping, C., Lu, H., Bin, C.: Research and implementation of ontology automatic construction based on relational database. In: Computer Science and Software Engineering. IEEE Computer Society (2008)

22. Kumova, B.I.: Symmetric properties of the syllogistic system inherited from the square of opposition (in review) (2015)

23. Kumova, B.I., Çakır, H.: Algorithmic decision of syllogisms. In: García-Pedrajas, N., Herrera, F., Fyfe, C., Benítez, J.M., Ali, M. (eds.) IEA/AIE 2010, Part II. LNCS (LNAI), vol. 6097, pp. 28-38. Springer, Heidelberg (2010)

24. Kumova, B.I., Çakir, H.: The fuzzy syllogistic system. In: Sidorov, G., Hernández Aguirre, A., Reyes García, C.A. (eds.) MICAI 2010, Part II. LNCS (LNAI), vol. 6438, pp. 418-427. Springer, Heidelberg (2010)

25. Laskey, K.B.: MEBN: A language for first-order bayesian knowledge bases. Artificial Intelligence 172 (2007)

26. Lin, L., Xu, Z., Ding, Y.: Owl ontology extraction from relational databases via database reverse engineering. Journal of Software 8(11) (2013)

27. Lubyte, L., Tessaris, S.: Automatic extraction of ontologies wrapping relational data sources. In: Bhowmick, S.S., Küng, J., Wagner, R. (eds.) DEXA 2009. LNCS, vol. 5690, pp. 128-142. Springer, Heidelberg (2009)

28. Lukasiewicz, T., Straccia, U.: Managing uncertainty and vagueness in description logics for the semanticweb. Web Semantics: Science, Services and Agents on the World Wide Web 6 (2008)

29. Maedche, A., Staab, S.: Ontology learning for the semantic web. IEEE Intelligent Systems and Their Applications 16(2) (2005)

30. Martinez-Cruz, C., Blanco, I.J., Vila, M.A.: Ontologies versus relational databases: are they so different? A comparison. Artificial Intelligence Review 38 (2011)

31. Motik, B., Shearer, R., Horrocks, I.: Hypertableau reasoning for description logics. Journal of Artificial Intelligence Research 36 (2009)

32. Myroshnichenko, I., Murphy, M.C.: Mapping er schemas to owl ontology. Semantic Computing, Berkeley (2009)

33. Park, C.Y., Laskey, K.B., Costa, P., Matsumoto, S.: Multi-entity bayesian networks learning in predictive situation awareness. In: International Command and Control Research and Technology Symposium (ICCRTS), US DoD (2013)

34. Qi, G., Pan, J.Z., Ji, Q.: A possibilistic extension of description logics. Description Logics (DL), Sun SITE Central Europe (CEUR) (2007)

35. Riguzzi, F.: Probabilistic description logics under the distribution semantics. Semantic Web Journal, SWJ (2013)

36. Rosati, R., Almatelli, A.: Improving query answering over DLLite ontologies. In: Principles of Knowledge Representation and Reasoning (KR). AAAI (2010)

37. Sahoo, S.S., Halb, W., Hellmann, S., Idehen, K., Thibodeau Jr., T., Auer, S., Sequeda, J., Ezzat, A.: A survey of current approaches for mapping of relational databases to rdf. W3C RDB2RDF Incubator Group (2009)

38. Santoso, H.A., Haw, S.C., Abdul-Mehdi, Z.T.: Ontology extraction from relational database: Concept hierarchy as background knowledge. Knowledge-Based Sys. (2011)

39. Sirin, E., Parsia, B., Cuenca-Grau, B., Kalyanpur, A., Katz, Y.: Pellet: A practical owldl reasoner. Journal of Web Semantics 5(2) (2007) 
40. Stoilos, G., Simou, N., Stamou, G., Kollias, S.: The fuzzy description logic fshin. In: Uncertainty Reasoning for the Semantic Web, CEUR Electronic Workshop (2005)

41. Stoilos, G., Simou, N., Stamou, G., Kollias, S.: Uncertainty and the semantic web. IEEE Intelligent Systems 21, 5 (2006)

42. Straccia, U.: Reasoning within fuzzy description logics. Journal of Artificial Intelligence Research 14 (2001)

43. Straccia, U.: SoftFacts: A top-k retrieval engine for ontology mediated access to relational databases. In: Systems, Man and Cybernetics (SMC). IEEE (2010)

44. Trinkunas, J., Vasilecas, O.: Building ontologies from relational databases using reverse engineering methods. In: Computer Systems and Technologies. ACM (2007)

45. Xu, J., Li, W.: Using relational database to build owl ontology from xml data sources. In: Computational Intelligence and Security Workshops. IEEE Computer Society (2007)

46. Yaguinuma, C.A., Magalhães Jr., W.C.P., Santos, M.T.P., Camargo, H.A., Reformat, M.: Combining fuzzy ontology reasoning and mamdani fuzzy inference system with hyFOM reasoner. In: Hammoudi, S., Cordeiro, J., Maciaszek, L.A., Filipe, J. (eds.) ICEIS 2013. LNBIP, vol. 190, pp. 174-189. Springer, Heidelberg (2014)

47. Zadeh, L.A.: Fuzzy logic and approximate reasoning. Syntheses 30 (1975)

48. Zadeh, L.A.: Syllogistic reasoning in fuzzy logic and its application to usuality and reasoning with dispositions. IEEE Transactions on Systems, Man and Cybernetics 15(6) (1985)

49. Zarechnev, M., Kumova, B.I.: Ontology-based fuzzy-syllogistic reasoning. In: Industrial, Engineering and Other Applications of Applied Intelligent Systems (IEAAIE). LNCS. Springer (2015)

50. Zhang, F., Ma, Z.M., Yan, L., Cheng, J.: Construction of fuzzy OWL ontologies from fuzzy EER models: A semantics-preserving approach. Fuzzy Sets and Sys. $229(2013)$

51. Zhang, F., Ma, Z.M., Yan, L., Wang, Y.: A description logic approach for representing and reasoning on fuzzy object-oriented database models. Fuzzy Sets and Systems 186 (2012) 